\title{
New Definition for Leukoplakia and Reclassification of Leukoplakias as Separated Disease Entities
}

\author{
Abdullatef Nureddin* \\ Department of Oral Surgery, Oral Medicine and oral pathology, Tripoli University, Libya
}

Submission: December 14, 2017; Published: February 06, 2018

*Corresponding author: Abdullatef Nureddin, Department of Oral Surgery, Oral Medicine and oral pathology, Tripoli University, Libya, Tel: 00218914319554, Email: yaema2000@gmail.com

\begin{abstract}
It is in the fact that, a proper and accepted definition of leukoplakia is still controversial, because the term leukoplakia alone is not specific disease entity but a clinical term means a white patch. A far better nomenclature could be one which gives some indication about pathogenesis of the lesion and to some extent specific clinical and histopathological features. Based on several studies of leukoplakias, it might be possible to suggest a new definition for the term leukoplakia and to reclassify leukoplakias as a separated disease entities that may meet the requirements of clinicians to understand the clinical course and different histopathological stages of leukoplakias to avoid confusion and to establish correct diagnosis of every individual case as this will affect the management of the patient. There will be, of course, a revision in the future as new knowledge accumulates. This paper will bring forward data for further study of different leukoplakias and their stages toward malignant transformation.
\end{abstract}

Keywords: Definition; Leukoplakia; Disease entities; Reclassification; Malignant transformation

\section{Introduction}

The normal colour of oral mucosa is pinkish-red but the oral mucosa is not uniformly have this colour, it is dark red in vestibular mucosa and lighter in gingiva. This normal color results from visible light passing through the translucent superficial layers of the covering stratified squamous epithelium, striking the capillary bed and then being reflected again [1].

Therefore, any changes in or on the covering epithelium such as keratosis (presence of keratin in normally non-keratinized mucosa), hyperkeratosis (increase in thickness of keratin), acanthosis (increase in thickness of covering epithelium), intercellular edema or invading of candidal hyphae in some oral candidiasis will result in white-appearing lesion due to reflection of light on hydrated keratin, keratin debris and desquamated epithelial cells. Therefore, classification of white lesions of oral mucosa should be meaningful to the clinician to give convenient information for comparing different lesions affecting oral mucosa which have a common clinical appearance, as the white patch may be caused by a simple trauma to a specific infectious disease or the underlying cause may be unknown [1-3].

White patches can be classified or grouped into many ways either clinically or histopathologically. Clinically, into lesions that cannot be scrapped off or wiped away by tongue blade which denote for keratotic lesions of oral mucosa and those lesions that can be scrapped off or wiped away by tongue blade, which denote for lesions resulted from sloughing, pseudo membranous necrotic tissue, necrotic keratinous debris or retention of desquamated epithelial cells of the covering epithelium. Although, white patches also being classified according to the etiology where this classification is the most common classification used in dentistry. This classification includes hereditary white patches, frictional keratosis, infective white patches, idiopatheic white patches, dermatological white patches and neoplastic white patches [1-3].

In contrast to clinical basis, histo pathologically, the classification of white patches depends on the microscopical features of epithelial dyasplasia where the lesion may shows $[1,2]$.

1. Mild dysplastic changes located in the lower one third of the covering epithelium.

2. Moderate dysplastic changes located in the lower two third of the covering epithelium.

3. Sever dysplastic changes (top-to-bottom dysplastic changes), also called carcinoma in situ.

4. Those lesions that show infiltrating squamous cell carcinoma.

5. Those lesions that show histopathological features of other diseases and conditions.

\section{Definition of leukoplakia}

The term "leukoplakia" was first applied by Karl Freiherr in 1861, who used it to describe white patches of the urinary tract 
[4], followed by Schwimmer in 1877, who first used the term to describe a white patch of the oral mucosa [5,6]. In 1930, studies on experimental animals concluded that leukoplakia can be induced in rabbits when subjected to tobacco smoke for 3 minutes per day [7]. In 1961, many studies showed that leukoplakia can occur in other mucous membranes such as rectum, vagina, uterus, vulva, paranasal sinuses, gallbladder, esophagus, eardrums, and pharynx [4].

Although, the word leukoplakia derived from Greek language, leucos means white and plakos means patch [6,8], therefore, leukoplakia in a simple definition means a white patch, but since many decades ago until now, the term leukoplakia is used by clinicians to denote for a white firm plaque that cannot be scraped off with a tongue blade and has tendency for malignant transformation. So it would be within this term of reference, leukoplakia was incorrectly used as a synonymous for any white patch in the oral mucosa rather than to a specific disease entity which led to confusion, misdiagnosis, and gross mismanagement of the lesion [9].

However, leukoplakia defined by World Health Organization as a white patch or plaque that cannot be characterized clinically or histopathologically as any other disease. This definition was modified slightly at an international symposium in 1994 to a predominantly white lesion of the oral mucosa that cannot be characterized as any other definable lesion. Leukoplakia is therefore, a clinical diagnosis arrived by exclusion of all other white patches of oral mucosa. This rather a negative definition highlights the fact that the diagnosis of leukoplakia is one of exclusion [1].

It is in the fact, that a proper and accepted definition of leukoplakia is still contraversal, because the term leukoplakia alone is not a disease entity (it has no definite clinical and histopathological features), which makes the definition for such lesion a difficult task if not impossible. Therefore, it has been suggested that leukoplakia is an incorrect term since there is so much argument about its use [6] and some clinicians in dental practice avoid using it at all [5].

A far better nomenclature would be one which gives some indication of the nature of the lesion, such as aetiology, clinical or histopathological features and then the diagnosis can be achieved by combination of clinic-pathological effort to find out the final diagnosis.

Based on several studies of leukoplakias and previous definitions of international symposiums, might there is a possibility for me to put a definition for leukoplakia that may meet the requirements of the clinicians for a proper diagnosis. Therefore, Leukoplakia is a white firm patch or plaque used clinically in association with certain keratotic lesions of oral mucosa which have tendency for malignant transformation.

\section{General Clinical Features of Leukoplakia}

Oral leukoplakia is a common lesion which present clinically as a white, whitish-yellow or grey plaque that cannot be scrapped off by tongue blade. Leukoplakia affects wide age range and the lesion vary in size from small to large area involving the oral mucosa, it can occur anywhere in the oral mucosa but the most common frequent sites are the tongue, floor of the mouth, lower lip, alveolar ridge, palate and buccal mucosa, although the lesion may be solitary or there may be multiple plaques scattered through the mouth. Oral leukoplakia is more common in males than females particularly between 40-70 years of age, it vary greatly in shape and distribution, the borders may either distinct or indistinct and smoothly countered or ragged, homogenous or rough in appearance [1-3].

Leukoplakia is characteristically asymptomatic, but infrequently the patient may complain from pain, burning sensation and discomfort. Although, the lesion may be discovered during routine oral examination or in some instances, the patient may seek professional consultation as the lesion enlarges and becomes more obvious or changed in color. The early lesion is usually nonpalpable macule, later, particularly in longstanding or persistent form of leukoplakia the surface may become rough and raised, thus becoming speckled or verrucous type, this clinical difference in appearance of the lesion may represent stages of malignant transformation or may associated with high risk of malignant transformation especially when the lesion is located on the floor of the mouth, lateral surface of the tongue and retro-molar area [6,9]. Leukoplakia is more frequently seen in Carribbean population and Indian subcontinent where chewing habits and reverse smoking is a common traditional practice adding to that the patient with leukoplakia is usually of poor general health [9-11].

\section{Discussion}

The aetiology of oral leukoplakia is multifactorial, some factors are well identified such as tobacco, candidal species, Epstein Barr virus, bacteria, and some extracts of herbal plants. Therefore, the correct diagnosis of leukoplakia should be associated with the aetiological factor rather than clinical appearance as many forms of leukoplakia can share similar clinical features.

\section{Tobacco smoking}

Tobacco smoking is widely practiced worldwide and considered as the most common cause of leukoplakia on oral mucosa. Several epidemiological studies cross the world have shown increase incidence of leukoplakia among smokers than in non-smokers due to high toxic agents of tobacco with ammonia, hydrogen cyanide and phenol. It is estimated that more than $80 \%$ of patients presented clinically with leukoplakia are smokers $[8,12]$.

Tobacco smoking may practiced as cigarette, cigar, pipe smoking, reverse smoking, bidi smoking or as chewing tobacco and snuff dipping, in cigarette smoking, the lesion is mostly diffuse and seen mainly on cheek, lip and tongue where in pipe and cigar smoking the lesion is usually manifested on the lip. In bidi smoking which made by rolling of dried and tender piece of leaf with locally grown tobacco, the lesion is usually present on the labial commissure, while in reverse smoking where the burning end of 


\section{Advances in Dentistry \& Oral Health}

cigarette is held within the oral cavity, the lesion is usually seen in the area of oral mucosa that directly irritated by the burning end Smokeless tobacco may be used as snuff (powdered tobacco) to being inhaled or as snuff-dipping which placed in labial or buccal sulci and tobacco chewing where also placed in the buccal sulcus and kept in the mouth for a long time, therefore, the lesion is usually seen in the buccal sulcus and mucobuccal fold close to the premolars and molars [1-3].

Pan chewing habit is one of the most widespread habits in the world particularly in the South-East Asia and India. Pan chewing habit which traditionally called mawa consists of a mixture of sun cured areca nut on a cellophane paper with tobacco flacks added and sprinkling of a few drops of lime prepared from Shell Sea. The cellophane paper with the mixture is then tied as a round ball and given to the customer. Mawa is practiced due to widespread believe that it satisfies hunger, causes euphoria and makes breath smell good, this habit induces leukoplakia and oral submucous fibrosis due to the action of some substances released by pan chewing that stimulate collagen synthesis, release carcinogenic alkaloids and nitrosamines $[1,7,8]$

Several studies have demonstrated a dose-response relationship between tobacco usage and oral leukoplakia as well as the decrease in the incidence of oral leukoplakia after tobacco smoking and smokeless tobacco cessation [13,14]. Although, several studies have shown a significant increase in keratinized cells of the tongue and hard palate mucosa in tobacco smokers individuals who clinically have healthy oral mucosa This preclinical alterations or changes have been reported on the results from smear and cytological examination of oral mucosa in male and female smokers and nonsmokers $[15,16]$. Adding to that, recent studies demonstrated that oral leukoplakia associated with tobacco smoking showed microscopically, the presence of chevron peaks in the keratin of the covering epithelium and incontinence of melanin pigmentation in the connective tissue core (lamina propria) which concluded to be a specific histo pathological feature and is highly suggestive of tobacco smoking as an important aetiological factor [1].

However, several previous and recent studies of tobacco and leukoplakia in different parts of the world concluded that there is strong evidence and strong relationship between tobacco usage of all types and the development of oral leukoplakia. Gayford and Haskell [3] concluded that if the term leukoplakia is to be used there must be clear distinction as to its meaning. The indiscriminate use of this term as synonymous for a white patch in the mouth, an area of keratosis, a premalignant lesion or a histological entity can lead only to confusion, a far better nomenclature would be one which gives some indication of the nature of the lesion and its aetiology. Therefore, it would be better and more appropriate if the lesion called tobacco-associated leukoplakia and regarded as a specific disease entity as the causative factor (various types tobacco usage) can be assessed clinically, although the lesion has some distinctive histological features discussed above.

\section{Candida Species}

Candida species principally candida albicans is one of the most frequent fungal infections affecting oral mucosa. It is a normal inhabitant in the oral cavity, throat, large bowel and vagina, although the carriage rate increased in pregnancy, tobacco smooking, denture wearers and presence of any medical conditions.

Most candida species are dimorphic fungi which can grow either as a hyphae (long filaments) or as yeast (grow by budding), therfore it is presumed that candida has a direct aetiological relationship with a lesion if hyphae are present in smear or histopathological section of the lesion. The pathogenic mechanism of candida species infection is not fully understood, but candida species secreted a variety of enzymes such as proteinase, lipases and a number of toxins that enable hyphae to invade epithelium. Candidal antigens stimulate an immunoresponse and induce delayed-type hypersensitivity reaction leading to tissue injury [1]

Candidal leukoplakia is also referred to as a Chronic hyperplastic candidosis. Candidal leukoplakia presents clinically as a keratotic rough dense white plaque of irregular thickness and density that can not be wiped away. In some lesions, erythematous areas may be seen within the white plaque giving a speckled appearance. Buccal mucosa near to the commissure of the lips is the most common site frequently affected in which the shape of the lesion may appears as triangular tapering posteriorly and may be associated with angular chelitis. Other parts of oral mucosa are less frequently involved. Although in many cases it is indistinguishable clinically from other types of leukoplakias [1-3].

Cawson [20], who first defined the lesion, considers the candidal hyphae as primary aetiological factor of epithelial hyperplasia and Norman et al. [9] concluded that some of these lesions have seem to result primary from the candida because they disappear after repeated topical application of nystatin cream Microscopically, candidal leukoplakia differs from other types of leukoplakias in that the covering stratified squamous epithelium is always parakeratinized and there is gross acanthosis of prickle cell layer with considerable intercellular oedema, acute inflammatory cell infiltrate and pseduepitheliomatous hyperplasia. The underlying connective tissue core shows intense acute and chronic inflammatory infiltration and a variable number of neutrophil leucocytes collecting toghter as microabscesses. In some cases differentiation from other types of leukoplakias is difficult. Therefore, the diagnosis of candidal leukoplakia (chronic hyperplastic candidosis) can be confirmed using PAS stain (special stains for candidal hyphae), so such a lesion may only be diagnosed on biopsy using PAS stain $[1,2,3,17]$,

\section{Epstein Barr Virus}

Hairy Leukoplakia (HL) is well documented separated disease entity which is strongly associated HIV infection, although it occurs also in non-HIV infected patiets receieving immunosuppressive therapy or those patients who have other immunodeficiency 
states. HL is caused by Epstein Barr Virud (EBV) and prsents clinically as a keratotic white patch with vertical folds or raised, corrugated or hairy surface on the lateral border of the tongue that cannot wiped away. The lesion may be seen on other parts of oral mucosa and the surface of the lesion may be smooth [18].

The diagnosis of hairy leukoplakia is usually simple at clinical examination particularly in a patient who is known to be HIV seropositive, but in difficult cases with no known cause, demonstration of EBV by serological tests is essential for diagnosis. Histopathologically, hairy leukoplakia shows features of virally infected cells such as swollen or ballon cells degeneration with prominent cell borders which seen as a band in the prickle cell layer and some cells show small darkly staining nuclei with perinuclear vacules due to displacement of chromatin to the periphery of the cells by EBV multiplication $[9,18,19]$.

As the diagnosis of viral infections of oral mucosa is based mainly on history, clinical examination and laboratory investigations such as:

1. Isolation of virus from the lesion and cultivated in tissue culture, fertilized eggs or laboratory animal.

2. Histopathological changes may be found in tissue using light or electron microscope. Viral antigens may be demonstrated using immunofluorescent, immunocytochemical or in-situ hybridization techniques.

3. Serological tests can be used for demonstration of antibodies by ELISA or immunoblotting techniques

4. Demonstration a significant rise in antibodies titre $[9,19]$.

Therefore, the final diagnosis of hairy leukoplakia can be achieved at the bases of clinico-pathological investigations.

\section{Bacteria}

Syphilis is the most severe treponemal disease which caused by a delicate spiral filament or spirochaete called treponema pallidum. Syphilis is almost always aquired venerealy but may be transmitted by blood transfusion and direct contact. Once treponema pallidum penetrates the skin, it spreads through blood stream within hours and becomes disseminated long before any local manifestation appears. Therefore, it is suggested that the first signs (formation of chancre) is due to hypersesitivity reaction at the site of entry as result of formation of sensitizing antibodies. At a later stage the other tissues become sensitized with development of generalized lesions of secondary syphilis.

Chancre is usually appears on the genital region and around the anus in homosexuals 2-4 weeks after infection, but may present on the oral mucosa such as lips or tip of the tongue. Chancre is indurated painless papule which breaks down to form ulcer which is typically described as a clean-based painless, shallow ulcer associated with regional lymphadenopathy. The chancre heals spontaneously over a period of a few weeks and secondary lesions develop about 6 weeks later, some 2-3 months after the initial exposure. This stage is characterized by generalized widespread non-pruritic skin rash (erythematous papules). In the past, the name given for this stage was (great pox).

The final stage may appear 2-3 years after the initial exposue which characterized by localized destruction ( formation of gumma) or diffuse inflammatory lesions. Gumma is a tumourlike mass or swelling which may be seen in the tongue or palate. The gumma may ulcerate to form a painless ulcer. Other oral manifestation of this stage including syphilitic leukoplakia and atrophic glossitis which may occur as result of endarthritis obliterans. Histopathologically a gumma is formed from a central area of coagulative necrosis surrounded by a zone of epithelioid cells, giant cells, lymphocytes, plasma cells and fibroblasts [19].

Cawson et al. [20] and Nevill et al. [8], concluded that the histopathological diagnosis of syphilitic leukoplakia is nonspecific and the diagnosis is confirmed by demonstrating the spiral organism by biopsy or dark-field examination of a smear or by other sensitive serological screening tests such as Venereal Disease Research Laboratory( VDRL) and the rapid plasma reagin (RPR), fluorescent treponemal antiboy absorption (FTA-ABS), T. pallidum hemagglutination assay (TPPA) and microhemagglutination assay for antibody to T. pallidum (MHA-TP) [8,20].

\section{Sanguinaria}

Sanguinaria is a substance extracted from herbal plant called (bloodroot) consisting benzophenanthidine alkaloids mixed with Viadent and used in mouthwashes and toothpaste products since 1982 because these products have shown to be effective in plaque control. Later, several studies have shown that this type of mouth wash when used for a period extended from 6 months to 12 years is strongly associated with leukoplakia in the vestibule of the mouth termed (sanguinaria associated keratosis or sanguinaria -induced leukoplakia). Clinically, sanguinaria-associated leukoplakia typically affects middle-age group and presented as a wrinkled or corrugated well-defined white lesion that cannot be wiped away in maxillary or mandibular vestibular mucosa. Histopathologically, several studies demonstrated that the lesion shows hyperkeratosis and dysplastic changes at the lower onethird of the stratified squamous epithelium such as basal cell hyperplasia, nuclear hyperchromatism and alteration in nuclearcytoplasmic ratio $[8,21]$.

From the relevant data, the diagnosis of sanguinariaassociated leukoplakia depends mainly on a good history and clinical examination while the distinctive histopathological features that noted in the lower third of the covering epithelium can added further support to the clinical diagnosis.

\section{Tendency for malignant transformation of oral leukoplakia}

Epidemiological studies have shown that leukoplakias are the most premalignant lesions of oral mucosa and the incidence of malignant transformation is variable, but leukoplakias presented on the ventral surface of the tongue, floor of the mouth and the 
lingual aspects of lower alveolar mucosa are high-risk sites. It is estimated that, malignant transformation ranges between $0.3 \%$ to $18 \%$ in different parts of the world in which about $14 \%$ of malignant transformation may occur over a period of 20 years while $4 \%$ over a period of 10 years and $50 \%$ of cases in erythroplakia are carcinoma in-situ or invasive squamous cell carcinoma on initial biopsy $[1,5,7,8,20]$.

However, instead to abandon the term leukoplakia as it means a white patch and a disease of exclusion, many new types of leukoplakias have been described such as conventional leukoplakia, thin leukoplakia, thick leukoplakia, reversible leukoplakia, irreversible leukoplakia, homogenous leukoplakia, non-homogenous leukoplakia, granular leukoplakia, nodular leukoplakia, verrucous leukoplakia and speckled leukoplakia [1$4,8,9]$.

In the fact, those types of leukoplakias are not a disease entities but a clinical description which represent the histopathological changes toward malignant transformation. Changes in clinical appearance of leukoplakia has been first described by Norman et al. [9], who concluded that early lesion of leukoplakia is a nonpalpable macule then the surface may become rough and raised, thus becoming a speckled or verrucous type of leukoplakia.

These changes were described later in detail by Neville BW [8], who concluded that early lesion of leukoplakia is thin and appears as a flat or slightly elevated white plaque which may be somewhat translucent, fissured or wrinkled with sharp demarcated borders. Thin leukoplakia (reversible leukoplakia) may disappear or enlarge and progress to stage called homogenous leukoplakia (thick leukoplakia or irreversible leukoplakia) characterized by thick white plaque. Some lesions develop increase surface irregularities and called granular leukooplakia and then nodular and verrucous leukoplakia. Some lesions show scattered reddish areas called erythroplakia or speckled leukoplakia which represent advanced dysplastic changes on biopsy. The stages of malignant transformation are summarized by Neville BW [8] as the following:

\section{Normal epithelium}

2. Thin smooth leukoplakia, shows hyperkeratosis and acanthosis

3. Thick fissured leukoplakia shows hyperkeratosis, acanthosis and mild dysplasia

4. Granular-verruciform leukoplakia shows irregular hyperkeratosis (verruciform hyperkeratosis) and moderate dysplastic changes

5. Erythroleukoplakia (speckled leukoplakia) shows irregular hyperkeratosis, epithelial atrophy and severs dysplastic changes (carcinoma in situ)

The description above was supported by several studies about the role of genetic alteration due to loss of chromosomal material from specific areas as result of non-disjunction, deletion, translocation and cross-over between two homologous genes in the chromosome. This is called loss of heterozygosis ( $\mathrm{LOH})$, where the mechanism involves a progressive accumulation of 6-10 genetic alteration in the epithelial cells, although different alteration occur at different times from normal epithelium to dysplastic epithelium which leads to carcinoma in-situ and invading squamous cell carcinoma. Soames \& Southam [1] concluded that when $\mathrm{LOH}$ occurred at $9 \mathrm{p}$, the normal epithelium undergoes mild epithelial dysplastic change, then new accumulation of genetic alteration of $\mathrm{LOH}$ at $3 p$ and $17 \mathrm{p}$ leading to moderate epithelial dysplastic change, where in advanced stages, LOH occurred at 11p, 13p and $14 p$ leading to carcinoma in-situ and finally LOH at $4 q, 6 p$ and $8 p$ leading to invasive squamous cell carcinoma.

Based on the discussion above and the new definition of leukoplakia (leukoplakia is a white firm patch or plaque used clinically in association with certain keratotic lesions of oral mucosa which have tendency for malignant transformation). It appears that it is more appropriate for some leukoplakias to be classified as a separated disease entities depending on the aetiopathogenesis, clinical and histopathological features to give a basis for clinical differential diagnosis and to establish the correct final diagnosis and to consider other forms of leukoplakias as a clinical stages toward malignant transformation.

\section{Classification of leukoplakias as a separated disease entities}

1. Tobacco-associated leukoplakia

2. Candidal leukoplakia (chronic hyperplastic candidiasis)

3. Hairy leukoplakia

4. Syphilitic leukoplakia

5. Sanguinaria-associated leukoplakia

However, other white patches such as white sponge naevus, oral manifestation of genodermatoses, leukoedema, frictional keratosis, other types of candidosis, lichen planus, nicotinic stomatitis, oral submucous fibrosis and neoplastic lesions, fordyces granules, oral keratosis of renal failure, psoriasis, verrciform xanthoma and skin grafts are well documented and classified under hereditary, traumatic, infective, dematological, neoplastic and miscellaneous disorders of oral mucosa [1-3,8,9].

\section{Conclusion}

It is concluded in this paper that, it might be more appropriate to bring a new definition for the term leukoplakia and to reclassify leucoplakias as a separated disease entities in association to their pathogenesis and to discuss the histological stages toward malignant transformation rather than to describe or to invent several types of leukoplakias, as this may will bring further confusion which can affect the diagnosis and treatment of the lesion. 


\section{Acknowledgment}

I would like to acknowledge all the references in this paper. Those famous names in oral and maxillofacial pathology are my teachers and my colleagues and I hope that I participate some work with them.

\section{References}

1. Soames JV, Southam JC (2007) Oral Pathology ( $4^{\text {th }}$ edn), Chapter 9, p. 119-127.

2. Malcolm A (1977) Burket's Oral Medicine. ( $7^{\text {th }}$ edn), Chapter 6, p. 6696.

3. Gayford JJ, Haskell R (1979) Clinical Oral Medicine. White Lesions of Oral Mucosa p. 72.

4. Petrou Steven P, David M Pinkstaff, Kevin J Wu, Kenneth J Bregg (2003) Leukoplakia of the Bladder. Cliggott Publishing, USA.

5. Tanaka Takuji, Tanaka Mayu, Tanaka Takahiro (2011) Oral Carcinogenesis and Oral Cancer Chemoprevention: A Review. Patholog Res Int 2011: 431246.

6. Odell W (2010) Clinical problem solving in dentistry ( $3^{\text {rd }}$ edn), Churchill Livingstone. Edinburgh, UK, pp. 209-217.

7. Roffo AH (2013) The carcinogenic effects of tobacco. World Health Organization.

8. Neville BW, Damm DD, Allen CM, Bouquot JE (2002) Oral \& maxillofacial pathology ( $2^{\text {nd }}$ edn), W.B. Saunders, Philadelphia, pp. 337-345.

9. Norman K Wood, Paul W Goaz (1980) Differential Diagnosis of Oral Lesions. ( $\left.2^{\text {nd }} e d n\right)$, Chapter 5, p. 70.

10. Greenberg MS, Glick M (2003) Burket's oral medicine diagnosis \& treatment $\left(10^{\text {th }}\right.$ edn), BC Decker. Hamilton, Ont, USA, pp. 87, 88, 9093,101-105.

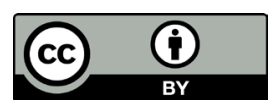

This work is licensed under Creative Commons Attribution 4.0 Licens DOI: 10.19080/ADOH.2018.07.555717
11. Soames JV, Southam JC (1999) Oral pathology (3 ${ }^{\text {rd }}$ edn), [Nachdr.]. (edn). Oxford [u.a.]: Oxford Univ. Press. pp. 139-140, 144-151.

12. Arduino PG, Bagan J, El-Naggar A, Carrozzo M (2013) Urban legends series: oral leukoplakia: Oral Dis 19(7): 642-659.

13. Banoczy J, Rigo O (1991) Prevalence study of oral precancerous lesions within a complex screening system in Hungary. Community Dent Oral Epidemiol 19(5): 265-267.

14. Banoczy J (1977) Follow-up studies in oral leukoplakia. J Maxillofac Surg 5(1): 69-75.

15. Banoczy J (1962) Observations sur l'alteration de la cornification de la muqueuse buccale sous linfluence du tabac. Bull Group Int Rech Sci Stomatol 5: 543-553.

16. Meyer J, Rubinstein AS, Medak H (1970) Early effects of smoking on surface cytology of the oral mucosa. Oral Surg Oral Med Oral Pathol 30(5): $700-710$.

17. Sitheeque MA, Samaranayake LP (2003) Chronic hyperplastic candidiasis/candidiasis (candidal leukoplakia). Crit Rev Oral Biol Med 14 (4): 253-267.

18. Coogan MM, Greenspan J, Challacombe SJ (2005) Oral lesions in infection with human immunodeficiency virus. Bull World Health Organ 83(9): 700-706.

19. Scully C (2008) Oral and maxillofacial medicine: the basis of diagnosis and treatment ( $\left.2^{\text {nd }} e d n\right)$, Churchill Livingstone, Edinburgh, UK pp. 216, $308,310-312$.

20. Cawson RA, Odell EW, Porter S (2002) Cawson essentials of oral pathology and oral medicine. ( $7^{\text {th }}$ edn), Churchill Livingstone, Edinburgh, UK, pp. 221-238.

21. (2006) Leukoplakia hosted by the American Academy of Oral and Maxillofacial Pathology.

\section{Your next submission with Juniper Publishers will reach you the below assets}

- Quality Editorial service

- Swift Peer Review

- Reprints availability

- E-prints Service

- Manuscript Podcast for convenient understanding

- Global attainment for your research

- Manuscript accessibility in different formats

( Pdf, E-pub, Full Text, Audio)

- Unceasing customer service

Track the below URL for one-step submission https://juniperpublishers.com/online-submission.php 